\title{
Characterization of the Influence of Grain Boundary Character on Oxidation of Nickel Using Atom Probe Tomography
}

\author{
Shery L. Welsh and Jeffrey L. Evans \\ University of Alabama in Huntsville, Materials Science Program
}

Nickel-based superalloys are used in many high temperature applications where there are considerable cyclic loads and dwell times such as turbine engines, various components of land-based power systems and nuclear reactor materials [1]. Therefore, improving the performance of this material to better withstand these environments is key. This study is focused on Nickel 200 and the effects of the grain boundary character distribution (GBCD) on oxidation at and near the crack tip during elevated temperature creep-fatigue conditions. Grain boundaries can broadly be categorized as "random" or "special". It has been shown that a higher fraction of special grain boundaries in a material does indeed provide resistance against fatigue damage, creep deformation and corrosion [2]. There are some studies that suggest this mechanism is controlled more by the connectivity of the random grain boundary network. Atom Probe Tomography can be used to analyze the detailed structure and chemistry of metal alloys at the atomic level [1] and is used in this study to further characterize the GBCD influence on oxidation.

Initially, three Nickel 200 specimens are cold rolled to a $3 \%, 6 \%$ and $18 \%$ reduction in thickness followed by a heat treatment at $800^{\circ} \mathrm{C}$ in argon for 26 minutes, 16 minutes, and 10 minutes, respectively, to obtain varying levels of special grain boundaries, random grain boundaries, and random grain boundary connectivity. These samples and one as-received sample are then mounted and polished for scanning electron microscopy and electron backscatter diffraction (SEM/EBSD) analysis where the types of grain boundaries and the level of connectivity can be mapped (see Figure 1) during post processing.

Once post-processing is complete, the samples are annealed in air at $1000^{\circ} \mathrm{C}$ for 30 hours for grain boundary oxidation analysis. A dual beam field emission SEM/FIB (focused ion beam) is used to cut out specific grain boundaries (random and special) for oxidation analysis in a 3D atom probe (see Figure 2 for sample results of another study).

3D atom probe tomography will allow for 3D analysis of the sample to include chemical identity and atomic position of the atoms. 3D atom probe tomography will shed light on how the GBCD influences the oxidation levels given the various parameters of cold rolling, heat treatment, and exposure to the environment, specifically oxygen.

The GBCD influence on oxidation of Nickel 200 from these test results will then be applied to other Nickel alloys. The results of this research will provide a better understanding of grain boundary 
oxidation of Nickel and Nickel alloys, which will help in elucidating the grain boundary embrittlement mechanism.

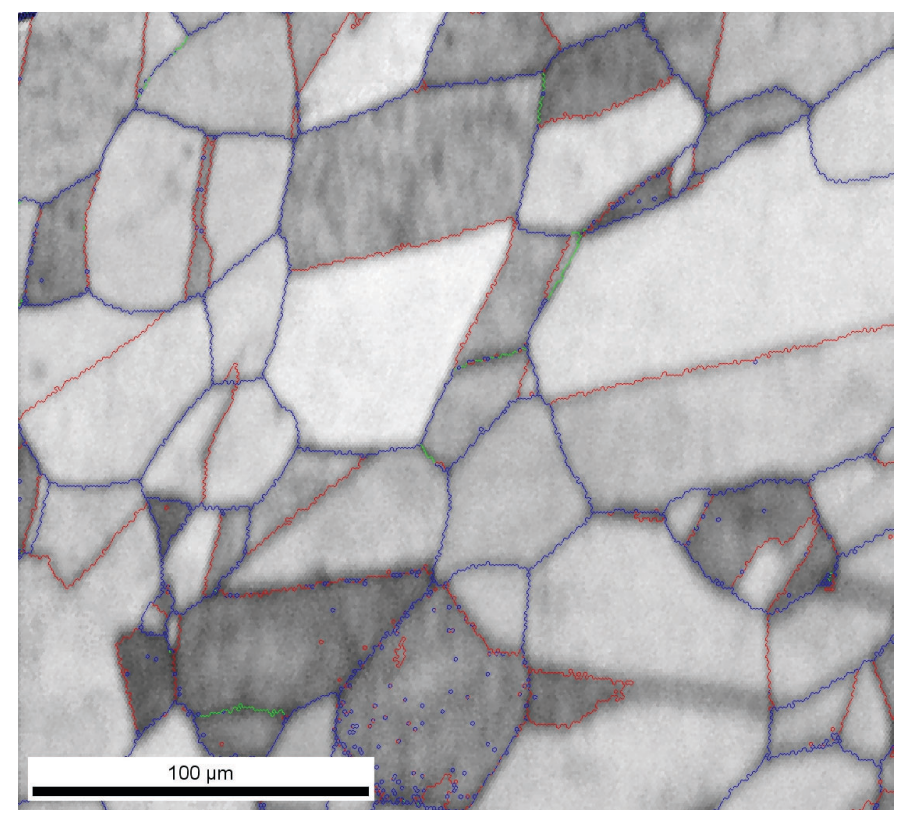

Figure 1 EBSD measurement of as-received Nickel 200 showing special (red) and random (blue) grain boundaries

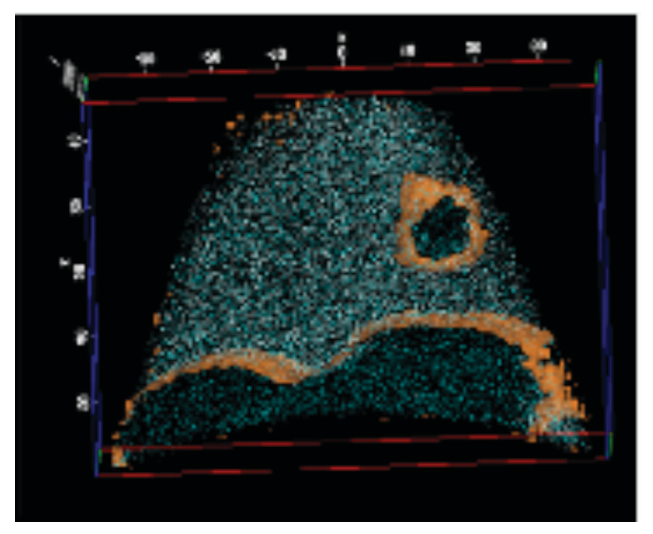

Figure 2 APT results: Copper precipitates in an Ag matrix (reproduced with permission, R.L. Martens)

The author would like to thank Ms. Olivia Underwood (University of Alabama in Huntsville) for her support and partnership in this research and Mr. Rich Martens (University of Alabama) for his atom probe tomography expertise.

\section{References}

[1] M. Olszta, D. Schreiber, and L. Thomas, Advanced Materials and Processes 170 (2012), pp. 1721

[2] T. Watanabe, Res Mechanica (1984), p. 47-84 